Pacific Journal of Mathematic 


\title{
ON THE IMBEDDABILITY OF THE REAL PROJECTIVE SPACES IN EUCLIDEAN SPACE ${ }^{1}$
}

\author{
W. S. MASSEY
}

1. Introduction. Let $P_{n}$ denote $n$-dimensional real projective space. This paper is concerned with the following question: What is the lowest dimensional Euclidean space in which $P_{n}$ can be imbedded topologically or differentiably? Among previous results along this line, we may mention the following;

(a) If $n$ is even, then $P_{n}$ is a non-orientable manifold, and hence cannot be imbedded topologically in $(n+1)$-dimensional Euclidean space, $R^{n+1}$.

(b) For any integer $n>1, P_{n}$ cannot be imbedded topologically in $R^{n+1}$, because its mod 2 cohomology algebra, $H^{*}\left(P_{n}, Z_{2}\right)$, does not satisfy a certain condition given by $\mathrm{R}$. Thom (see [6], Theorem V, 15).

(c) If $2^{k-1} \leqq n<2^{k}$ then $P_{n}$ cannot be imbedded topologically in Euclidean space of dimension $2^{k}-1$. This result follows from knowledge of the Stiefel-Whitney classes of $P_{n}$ (see Thom, loc. cit., Theorem III. 16 and E. Stiefel, [5]; also [4]).

In the present paper, we prove the following result: If $m=2^{k}, k>0$, then $P_{3 m-1}$ cannot be imbedded differentiably in $R^{1 m}$. For example $P_{5}$ cannot be imbedded differentiably in $R^{8}$, nor can $P_{11}$ be imbedded in $R^{16}$. Of course if $n>m, P_{n}$ cannot, a fortiori, be imbedded differentiably in $R^{4 m}$. Thus for many values of $n$ our theorem is an improvement over previous results on this subject. ${ }^{1}$

The proof of this theorem depends on certain general results on the cohomology mod 2 of sphere bundles. These general results are formulated in $\S 2$, and in $\S 3$ the proof of the theorem is given. Finally in $\S 4$ some open problems are discussed.

The author acknowledges with gratitude that he has benefited from several stimulating conversations with F. P. Peterson on this topic.

2. Steenrod squares in a sphere bundle with vanishing characteristic. Let $p: E \rightarrow B$ be a locally trivial fibre space (in the sense of

Received December 29, 1958. During the preparation of this paper, the author was partially supported by a grant from the National Science Foundation. An abstract announcing the main result of this paper was submitted to the American Mathematical Society in December, 1958; see the Notices of the American Mathematical Society, 6 (1959), 61.

1 This result partially solves a problem proposed by S. S. Chern (see Ann. Math., 60 (1954), p. 222). It follows that $P_{n}$ cannot be imbedded in $R^{n+2}$ for $n>3$ except possibly in case $n=2^{k}-1, k>2$. The case $n=2^{k}-1$ is still open. The importance of this problem, and some of its implications, were emphasized by $\mathrm{H}$. Hopf in his address at the International Congress of Mathematicians held in Cambridge, Massachusetts in 1950. This address is published in volume I of the Proceedings of the Congress (see pp. 193-202). 
[6]) with fibre a sphere of dimension $k-1$. We will use the following notation (due to Thom) for the Gysin sequence with the integers mod 2, $Z_{2}$, as coefficients:

$$
\cdots \longrightarrow H^{q-k}(B) \stackrel{\mu}{\longrightarrow} H^{q}(B) \stackrel{p^{*}}{\longrightarrow} H^{q}(E) \stackrel{\psi}{\longrightarrow} H^{q-k+1}(B) \longrightarrow \cdots
$$

Recall that the homomorphism $\mu$ is multiplication by the mod 2 characteristic class: $\mu(x)=x \cdot w_{k}$ for any $x \in H^{q-k}(B)$. In case $w_{k}=0$, then $\mu=0$, and the Gysin sequence splits up into pieces of length three as follows :

$$
0 \longrightarrow H^{q}(B) \stackrel{p^{*}}{\longrightarrow} H^{q}(E) \stackrel{\psi}{\longrightarrow} H^{q-k+1}(B) \longrightarrow
$$

Moreover, if we choose an element $a \in H^{k-1}(E)$ such that $\psi(a)=1 \in H^{0}(B)$, then any element $x \in H^{q}(E)$ may be expressed uniquely in the form

$$
x=p^{*}(u)+a \cdot p^{*}(v)
$$

where $u \in H^{q}(B)$ and $v \in H^{q-k+1}(B)$ (the proof is the same as that given in $\S 8$ of [3] except that here we are using $Z_{2}$ for coefficients). It is clear from this formula that the Steenrod squares and cup products in $H^{*}(E)$ are completely determined provided we know the Steenrod squares and products in $H^{*}(B)$, and provided we can express $S q^{i}(a)$ for $1 \leqq i \leqq$ $k-1$ in the form (1), i.e., provided the cohomology classes $\alpha_{i} \in H^{h-1+i}(B)$ and $\beta_{i} \in H^{i}(B)$ in the expression

$$
S q^{i}(\alpha)=p^{*}\left(\alpha_{i}\right)+\alpha \cdot p^{*}\left(\beta_{i}\right)
$$

are known for $1 \leqq i \leqq k-1$. Of course the classes $\alpha$ and $\alpha_{i}$ are not uniquely determined. If $a^{\prime}$ is any other element of $H^{k-1}(E)$ such that $\psi\left(a^{\prime}\right)=1$, then by exactness of the Gysin sequence there exists an unique element $b \in H^{k-1}(B)$ such that

$$
a^{\prime}=a+p^{*}(b) \text {. }
$$

Corresponding to formula (2) there is an analogous formula

$$
S q^{i}\left(a^{\prime}\right)=p^{*}\left(\alpha_{i}^{\prime}\right)+a^{\prime} \cdot p^{*}\left(\beta_{i}^{\prime}\right) .
$$

An easy computation shows that

$$
\left\{\begin{array}{l}
\alpha_{i}^{\prime}=\alpha_{i}+b \beta_{i}+S q^{i}(b), \\
\beta_{i}^{\prime}=\beta_{i}
\end{array}\right.
$$

Thus $\beta_{i}$ is unique; it is an invariant of the given fibre space. However, only the coset of $\alpha_{i}$ modulo the subgroup $\left\{\beta_{i} b+S q^{i} b: b \in H^{k-1}(B)\right\}$ is unique. This coset is also an invariant of the given fibre space. 
Lemma. $\quad \beta_{i}=w_{i}$, the ith Stiefel-Whitney class mod 2 of the fibre space.

This lemma is due essentially to Liao, [2]. See also an analogous proof in Massey, [3], § 9 .

Thus $\beta_{i}$ is identified with a standard invariant of sphere spaces. On the other hand, the coset of $\alpha_{i}$ does not seem to be related to any standard invariants. It may be thought of as a sort of "secondary characteristic class", defined for all sphere spaces for which the mod 2 characteristic class $w_{k}$ vanishes.

In view of this lemma we may write the above equations in the following form:

$$
\begin{gathered}
S q^{i}(a)=p^{*}\left(\alpha_{i}\right)+a \cdot p^{*}\left(w_{i}\right) \\
\alpha_{\imath}^{\prime}=\alpha_{i}+b w_{i}+S q^{i}(b), \quad b \in H^{k-1}(B) .
\end{gathered}
$$

3. Application to the problem of imbedding manifolds in Euclidean space. Our method of applying the results of the preceding section to prove that certain manifolds cannot be imbedded differentiably in $r$-dimensional Euclidean space is essentially the same as that used in our earlier paper [3]. To save the reader the trouble of referring to this earlier paper, we give a brief summary of the essential points of this method.

Let $M^{n}$ be a compact connected differentiable manifold which is imbedded differentiably in an $(n+k)$-sphere, $S^{n+k}$. We assume that $S^{n+k}$ has been given a Riemannian metric. Choose a positive number $\varepsilon$ so small that given any point $a \in S^{n+k}$ of distance $\leqq \varepsilon$ from $M^{n}$, there exists a unique geodesic segment through a of length $\leqq \varepsilon$ normal to $M^{n}$. Let $N$ denote the set of all points $a \in S^{n+k}$ whose distance from $M^{n}$ is $<\varepsilon . \quad N$ is an open tubular neighborhood of $M^{n}$ in $S^{n+k}$. Let $E$ denote the boundary of $N$, and $p: E \rightarrow M^{n}$ the projection defined by assigning to each point $a \in E$ the point $p(a) \in M^{n}$ where the unique geodesic segment through $a$ of length $\varepsilon$ normal to $M^{n}$ meets $M^{n}$. Then $\left(E, p, M^{n}\right)$ is a realization of the normal $(k-1)$-sphere bundle of $M^{n}$ for the given imbedding, and $E$ is a hypersurface in $S^{n+k}$. Let $V$ denote the complement of $N$ is $S^{n+k}$, and let $j: E \rightarrow V$ denote the inclusion map.

For convenience we introduce the following notation: $A^{q}$ denotes the image of the homomorphism $j^{*}: H^{q}(V, G) \rightarrow H^{q}(E, G)$, where $G$ is the coefficient ring, and $A^{*}=\sum_{q} A^{q}$. Then $A^{*}$ and $p^{*}\left[H^{*}\left(M^{n}, G\right)\right]$ are both sub-rings of $H^{*}(E, G)$, and they are obviously closed under any cohomology operations such as Steenrod squares and reduced $p$ th powers. Even more, $A^{*}$ and $p^{*}\left[H^{*}\left(M^{n}, G\right)\right]$ are " permissible sub-algebras" of $H^{*}(E, G)$ in the terminology of Thom, [6], p. 177. The sub-ring $A^{*}$ must satisfy the following conditions: 


$$
A^{\circ}=H^{\circ}(E, G)
$$

$$
H^{q}(E, G)=A^{u}+p^{*} H^{q}\left(M^{n}, G\right)
$$

$(0<q<n+1$, direct sum $)$

$$
A^{q}=0
$$

$$
\text { for } q \geqq n+k-1 \text {. }
$$

The proof that conditions (6), (7), and (8) hold is based on Theorem V.14 of Thom [6]; see also $\S 14$ of [3]. The existence of the sub-algebra $A^{*}$ satisfying these conditions is a rather stringent requirement on $H^{*}(E, G)$.

Our program for trying to prove that a certain manifold $M^{n}$ cannot be imbedded differentiably in $S^{n+k}$ (or equivalently, in Euclidean $(n+k)$ space, $R^{n+k}$ ) may be briefly outlined as follows. Assume that such an imbedding is possible, and let $p: E \rightarrow M^{n}$ denote the normal $(k-1)$ sphere bundle of this imbedding. By a well-known theorem of Seifert and Whitney, the characteristic class of the normal bundle vanishes, hence the Gysin sequence splits up into pieces of length three as described in the preceding section. Then if one can determine the structure of the cohomology ring of $E$ and perhaps determine some other cohomology operations in $E$, it may be possible to prove that $H^{*}(E, G)$ does not admit any sub-ring $A^{*}$ satisfying the conditions stated in the preceding paragraph. But this is a contradiction.

Using this method with $G=Z_{2}$, we will now prove our main result:

THEOREM. If $m=2^{k}, k>0$, then $P_{3 m-1}(R)$ cannot be imbedded differentiably in $R^{4 m}$.

Proof. Let $x$ be the generator or $H^{1}\left(P_{3 m-1}, Z_{2}\right)$. As is well known, the cohomology algebra $H^{*}\left(P_{3 m-1}, Z_{2}\right)$ is the truncated polynomial algebra generated by $x$ and subject to the sole relation $x^{3 m}=0$. According to a result of E. Stiefel [5], the total Stiefel-Whitney class $w=\sum_{i \leq 0} w_{i}$ of the tangent bundle of $P_{3 m-1}$ is given by the formula

$$
w=(1+x)^{3 m} \text {. }
$$

(This may be proved directly by the method of $\mathrm{Wu}$ [7].) Using the Whitney duality theorem, one sees that the total Stiefel-Whitney class $\bar{w}=\sum_{i \geqq 0} \bar{w}_{i}$ of the normal bundle is given by

$$
\bar{w}=(1+x)^{m}
$$

since

$$
w \bar{w}=(1+x)^{3 m}(1+x)^{m}=(1+x)^{4 m}=1+x^{4 m}=1
$$

(Recall that $m=2^{k}$ ). It follows that $\bar{w}_{1}=0$ and $\bar{w}_{m}=x^{m}$. Now assume that the differentiable imbedding of $P_{3 m-1}$ in $S^{4 m}$ is possible and let $p: E \rightarrow P_{3 m-1}$ denote the normal bundle, whose fibre is an $m$-sphere. 
The characteristic class $\bar{w}_{m+1}=0$, hence we can apply the method of $\S 1$. Choose $a \in H^{m}\left(E, Z_{2}\right)$ such that $\psi(a)=1$. Then equations (4) of $\S 2$ applied to this case give

$$
\begin{aligned}
S q^{1}(a) & =p^{*} \alpha_{1} \\
a^{2} & =S q^{m}(a)=p^{*} \alpha_{m}+a \cdot p^{*}\left(x^{m}\right) .
\end{aligned}
$$

If $a^{\prime} \in H^{m}\left(E, Z_{2}\right), \psi\left(a^{\prime}\right)=1$, and $a^{\prime} \neq a$, then of necessity $a^{\prime}=a+p^{*}(b)$ with $b=x^{m}$. Therefore equation (5) of $\S 2$ gives

$$
\begin{gathered}
\alpha_{1}^{\prime}=\alpha_{1}+S q^{1}\left(x^{m}\right)=\alpha_{1} \\
\alpha_{m}^{\prime}=\alpha_{m}+x^{m} \cdot x^{m}+S q^{m} x^{m}=\alpha_{m} .
\end{gathered}
$$

Hence $\alpha_{1}$ and $\alpha_{m}$ are invariants, independent of the choice of $a$. Since $H^{m}\left(E, Z_{2}\right)$ admits the direct sum decomposition

$$
H^{m}\left(E, Z_{2}\right)=A^{m}+p^{*}\left[H^{m}\left(P_{3 m-1}, Z_{2}\right)\right]
$$

by (7), it follows that we may choose the cohomology class $a$ so that it belongs to $A^{m}$. From now on we assume such a choice has been made. Note also that it follows directly from the Gysin sequence that for $q>0$.

$$
\operatorname{rank} A^{q}=\operatorname{rank} H^{q-m}\left(P_{3 m-1}, Z_{2}\right)
$$

where $A^{q}$ and $H^{q-m}\left(P_{3 m-1}\right)$ are considered as vector spaces over the field $Z_{2}$. Thus $\operatorname{rank} A^{q}=0$ or 1 for all values of $q$; it follows that $A^{q}$ has at most one non-zero element.

First, we consider the invariant $\alpha_{1}$. Two cases are possible: $\alpha_{1}=0$, or $\alpha_{1}=p^{*}\left(x^{m+1}\right)$. If $\alpha_{1}=p^{*}\left(x^{m+1}\right)$, then the sub-ring $A^{*}$ is not closed under the operation $S q^{1}$, which is already a contradiction. For the remainder of the proof we will assume that $\alpha_{1}=0$, i.e. $S q^{1} a=0$, and show that this also leads to a contradiction.

Next we consider the invariant $\alpha_{m}$. Here again two cases are possible, $\alpha_{m}=0$ or $\alpha_{m}=x^{2 m}$. First let us consider the case where $\alpha_{m}=$ 0 , i.e.., $a^{2}=a \cdot p^{*}\left(x^{m}\right)$. Since $a^{2} \neq 0$, it must be the unique non-zero element of $A^{2 m}$. Let $y$ denote the unique non-zero element of $A^{2 m-1}$. It is clear that either

$$
y=p^{*}\left(x^{2 m-1}\right)+a \cdot p^{*}\left(x^{m-1}\right) \text { or } y=a \cdot p^{*}\left(x^{m-1}\right) .
$$

Now $a^{2} y \in A^{4 m-1}$, therefore $a^{2} \cdot y=0$ by equation (8). An easy calculation shows that $a^{2}\left(a \cdot p^{*} x^{m-1}\right)=a \cdot p^{*}\left(x^{3 m-1}\right) \neq 0$. It follows that $y=$ $p^{*}\left(x^{2 m-1}\right)+a \cdot p^{*}\left(x^{m-1}\right)$. Next, a computation shows that

$$
S q^{1}(y)=S q^{1}\left[p^{*} x^{2 m-1}+a \cdot p^{*} x^{m-1}\right]=p^{*}\left(x^{2 m}\right)+a \cdot p^{*}\left(x^{m}\right) .
$$

Thus $S q^{1}(y)$ and $a^{2}$ are distinct non-zero elements of $A^{2 n}$ which is obviously impossible. Thus we see that the assumption $\alpha_{m}=0$ leads to 
a contradiction.

Next, consider the case where $\alpha_{m}=x^{2 m}$, i.e. $a^{2}=p^{*}\left(x^{2 m}\right)+a \cdot p^{*}\left(x^{m}\right)$. The pattern of the proof in this case is the same as in the preceding paragraph. Let $y$ be the unique non-zero element of $A^{2 m-1}$ as before, then either $y=p^{*}\left(x^{2 m-1}\right)+a \cdot p^{*}\left(x^{m-1}\right)$ or $y=a \cdot p^{*}\left(x^{m-1}\right)$, and we must have $a^{2} y=0$ exactly as before. Once again an easy calculation shows that $a^{2}\left(p^{*} x^{2 m-1}+a \cdot p^{*} x^{m-1}\right) \neq 0$, hence we must have $y=a \cdot p^{*} x^{m-1}$. Again, one finds that $S q^{1} y=a \cdot p^{*} x^{m} \neq a^{2}$, which is a contradiction. Thus we have shown that the assumption that $P_{3 m-1}$ can be imbedded differentiably in $S^{4 m}$ leads to a contradiction.

4. Some open problems. H. Hopf has proved in [1] that $P_{n}$ can be imbedded differentiably in $R^{2 n-1}$ or $R^{2 n}$, according as $n$ is odd or even, i.e., according as $P_{n}$ is orientable or not. This result, together with our main theorem and the previous results mentioned in the introduction, enables one to settle definitely the question of imbedding $P_{n}$ in the lowest possible dimensional Euclidean space for $n \leqq 5$ : for $n \leqq 5$, Hopf's result is the best possible. The first undecided case is $P_{6}$. It follows from Hopf's result that it can be imbedded in $R^{12}$, and from our result that it cannot be imbedded in $R^{8}$. Can it be imbedded in $R^{9}$ ?

The invariants $\alpha_{i}$ introduced in $\S 2$ raise many interesting questions. Are these invariants of the normal bundle the same for any imbedding of a manifold in Euclidean space? Or, is it possible to give an example of different imbeddings of a manifold in Euclidean space which give rise to different sets of invariants $\alpha_{i}$ for the corresponding normal bundles ?' In any case, it seems reasonable to hope that a further investigation of their properties may furnish new tools for proving non-imbeddability theorems for manifolds.

One may also carry out an analogous investigation using the integers $\operatorname{mad} p, Z_{p}$, for any odd prime $p$ as coefficients, and using Steenrod $p$ th powers instead of Steenrod squares.

\section{REFERENCES}

1. H. Hopf, Sysieme Symmetrischer Bilinsarformen und Eulkidische Modclle der Projektiven Räume, Vierteljschr. Naturf. Ges. Zürich, 85 (1940), (32), 165-177.

2. S. D. Liao, On the theory of obstructions of fiber bundles, Ann. of Math., 60 (1946), 146-191.

3. W. S. Massey, On the cohomology ring of a sphere bundle, Jour. Math. and Mech., 7 (1958), 265-290.

4. F. P. Peterson, Some non-embedding problems, Bol. Soc. Mat. Mex., 2 (1957), 9-15.

2 This question is related to the following: It is possible to give an example of an $n$ manifold $M^{n}$ which admits two different imbeddings in $R^{n+k}$ such that the normal bundles of these imbeddings are non-equivalent? As far as I know, the answer to this question is not known. 
5. E. Stiefel, Über Richtungsfelder in den Projektiven Räumen und einen Satz aus der Reelen Algebra, Comm. Math. Helv., 13 (1940), 201-218.

6. R. Thom, Espaces fibrés eu Spheres et Carrés de Steenrod, Ann. Ecole Norm. Sup., 69 (1952), 109-182.

7. W. T. Wu, Classes charactéristiques et i-carrés d'une variété, C. $\Lambda$. Acad. Sci. Paris, 230 (1950), 508-511.

BROWN UNIVERSITY 



\section{PACIFIC JOURNAL OF MATHEMATICS}

\section{EDITORS}

\section{David Gilbarg}

Stanford University Stanford, California

\section{R. A. Beaumont}

University of Washington

Seattle 5, Washington

\section{A. L. Whiteman}

University of Southern California Los Angeles 7, California

L. J. Paige

University of California

Los Angeles 24, California

\section{ASSOCIATE EDITORS}

E. F. BECKENBACH
C. E. BURGESS
E. HEWITT
A. HORN

A. HORN

\author{
V. GANAPATHY IYER \\ R. D. JAMES \\ M. S. KNEBELMAN \\ L. NACHBIN
}

I. NIVEN

T. G. OSTROM

H. L. ROYDEN

M. M. SCHIFFER
E. G. STRAUS

G. SZEKERES

F. WOLF

K. YOSIDA

\section{SUPPORTING INSTITUTIONS}

\author{
UNIVERSITY OF BRITISH COLUMBIA \\ CALIFORNIA INSTITUTE OF TECHNOLOGY \\ UNIVERSITY OF CALIFORNIA \\ MONTANA STATE UNIVERSITY \\ UNIVERSITY OF NEVADA \\ OREGON STATE COLLEGE \\ UNIVERSITY OF OREGON \\ OSAKA UNIVERSITY \\ UNIVERSITY OF SOUTHERN CALIFORNIA
}

\author{
STANFORD UNIVERSITY \\ UNIVERSITY OF TOKYO \\ UNIVERSITY OF UTAH \\ WASHINGTON STATE COLLEGE \\ UNIVERSITY OF WASHINGTON \\ * * * * \\ AMERICAN MATHEMATICAL SOCIETY \\ CALIFORNIA RESEARCH CORPORATION \\ HUGHES AIRCRAFT COMPANY \\ SPACE TECHNOLOGY LABORATORIES
}

Mathematical papers intended for publication in the Pacific Journal of Mathematics should be typewritten (double spaced), and the author should keep a complete copy. Manuscripts may be sent to any one of the four editors. All other communications to the editors should be addressed to the managing editor, L. J. Paige at the University of California, Los Angeles 24, California.

50 reprints per author of each article are furnished free of charge; additional copies may be obtained at cost in multiples of 50 .

The Pacific Journal of Mathematics is published quarterly, in March, June, September, and December. The price per volume (4 numbers) is $\$ 12.00$; single issues, $\$ 3.50$. Back numbers are available. Special price to individual faculty members of supporting institutions and to individual members of the American Mathematical Society: $\$ 4.00$ per volume; single issues, $\$ 1.25$.

Subscriptions, orders for back numbers, and changes of address should be sent to Pacific Journal of Mathematics, 2120 Oxford Street, Berkeley 4, California.

Printed at Kokusai Bunken Insatsusha (International Academic Printing Co., Ltd.), No. 6, 2-chome, Fujimi-cho, Chiyoda-ku, Tokyo, Japan.

PUBLISHED BY PACIFIC IOURNAL OF MATHEMATICS, A NON-PROFIT CORPORATION

The Supporting Institutions listed above contribute to the cost of publication of this Journal, but they are not owners or publishers and have no responsibility for its content or policies. 


\section{Pacific Journal of Mathematics}

\section{Vol. 9, No. $3 \quad$ July, 1959}

Errett Albert Bishop, A minimal boundary for function algebras . . . . . . . . . . . . 629

John W. Brace, The topology of almost uniform convergence . . . . . . . . . . . . 643

Cecil Edmund Burgess, Chainable continua and indecomposability .......... 653

L. Carlitz, Multiplication formulas for products of Bernoulli and Euler

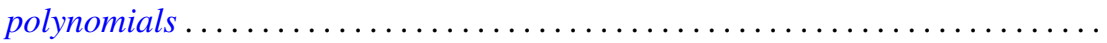

Eckford Cohen, A class of residue systems (mod $r$ ) and related arithmetical

functions. II. Higher dimensional analogues ....................

Shaul Foguel, Boolean algebras of projections of finite multiplicity . . . . . . . . . .

Richard Robinson Goldberg, Averages of Fourier coefficients .................

Seymour Goldberg, Ranges and inverses of perturbed linear operators .

Philip Hartman, On functions representable as a difference of convex functions ....

Milton Vernon Johns, Jr. and Ronald Pyke, On conditional expectation and

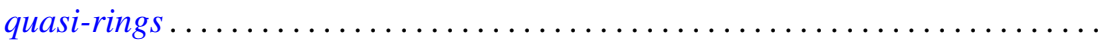

Robert Jacob Koch, Arcs in partially ordered spaces ....................

Gregers Louis Krabbe, A space of multipliers of type $L^{p}(-\infty, \infty) \ldots \ldots \ldots \ldots$

John W. Lamperti and Patrick Colonel Suppes, Chains of infinite order and their

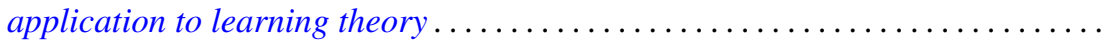

Edith Hirsch Luchins, On radicals and continuity of homomorphisms into Banach

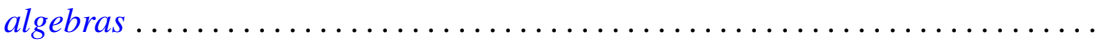

T. M. MacRobert, Multiplication formulae for the E-functions functions of their parameters.

Michael Bahir Maschler, Classes of minimal and representative domains and their kernel functions.

William Schumacher Massey, On the imbeddability of the real projective spaces in Euclidean space.

Thomas Wilson Mullikin, Semi-groups of class $\left(C_{0}\right)$ in $L_{p}$ determined by parabolic differential equations

Steven Orey, Recurrent Markov chains

Ernest Tilden Parker, On quadruply transitive groups ........ . .

Calvin R. Putnam, On Toeplitz matrices, absolute continuity, and unitary

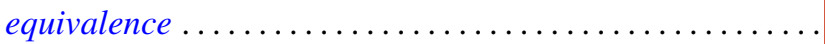

Helmut Heinrich Schaefer, On nonlinear positive operators.

Robert Seall and Marion Wetzel, Some connections between continued fractions and convex sets

Robert Steinberg, Variations on a theme of Chevalley

Olga Taussky and Hans Zassenhaus, On the similarity transformation between a

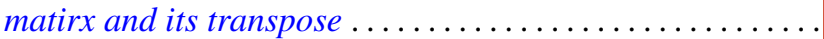

Emery Thomas, The suspension of the generalized Pontrjagin cohomology

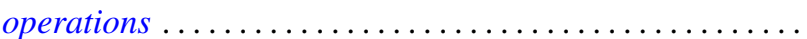

Joseph L. Ullman, On Tchebycheff polynomials ..................... 913

Richard Steven Varga, Orderings of the successive overrelaxation scheme ........ 925

Orlando Eugenio Villamayor, Sr., On weak dimension of algebras . 